\title{
As Estratégias de Aprendizagem e a Produção de Textos Narrativos
}

\author{
Learning Strategies and Narrative Text Production
}

\author{
Elis Regina da Costa $^{*}, a$ \& Evely Boruchovitch ${ }^{b}$ \\ ${ }^{a}$ Universidade Federal de Goiás \\ ${ }^{b}$ Universidade Estadual de Campinas
}

\begin{abstract}
Resumo
O objetivo da presente pesquisa foi avaliar a eficácia de uma intervenção em estratégias de aprendizagem na melhoria da qualidade da produção de textos de alunos. $\mathrm{O}$ estudo baseou-se num delineamento quase-experimental, realizado em 3 etapas: pré-teste, intervenção e pós-teste. A qualidade da produção textual foi avaliada por meio da comparação de dois textos narrativos (pré e pós-teste), quanto aos aspectos estruturais (presença de elementos básicos), ao nível de articulação de idéias (categorias hierárquicas), aos erros ortográficos e à quantidade de linhas escritas. Participaram do estudo 35 alunos da $6^{\text {a }}$ série de uma escola pública da cidade de Catalão, Goiás, que foram distribuídos aleatoriamente nos grupos experimental $(N=18)$ e controle $(N=17)$. Constatou-se que os alunos do grupo experimental produziram textos, no pós-teste, de melhor qualidade, caracterizados por uma estrutura narrativa adequada, idéias mais bem articuladas e maior quantidade de linhas escritas.

Palavras-chave: Estratégias de aprendizagem; Produção de textos; Formação de professores; Narrativas.

Abstract

The purpose of this research was to evaluate the efficacy of a learning strategy intervention for the improvement of quality in narrative production. It was based on a quasi-experimental design with three stages: pretest, intervention sessions, and post-test. The quality of text production was examined by comparing two narratives (pre and post-test) in relation to text structure (presence of basic elements), level of idea articulation (hierarchy categories), spelling mistakes and number of written lines. The sample consisted of $356^{\text {th }}$ grade students from a public school in Catalão-GO who were randomly distributed to experimental $(N=18)$ and control group $(N=17)$. The results showed that experimental group students produced better narratives in post-test characterized by adequate structure, good articulation of ideas and more written lines.

Keywords: Learning strategies; Text production; Teacher formation; Narratives.
\end{abstract}

Diversas investigações apontam uma relação entre o uso adequado de estratégias de aprendizagem e um bom desempenho acadêmico (Lopes, 1997; Kieft, Rijlaarsdam, \& Van den Bergh, 2006; Torrance, Fidalgo, \& Garcia, 2007). De acordo com Nisbett, Schucksmith e Dansereau (1979), citados por Pozo (1995), estratégias de aprendizagem podem ser entendidas como atividades ou procedimentos utilizados com o propósito de facilitar a aquisição, o armazenamento e/ou a utilização da informação. Outros autores a definem como processos conscientes, controlados pelos estudantes para atingirem objetivos de aprendizagem, bem como qualquer procedimento adotado para a realização de uma determinada tarefa (Da Silva \& De Sá, 1997).

Muitos estudantes não sabem como resumir, selecionar as idéias principais de um texto ou mesmo como estudar

*Endereço para correspondência: Rua Coronel Quirino, 910,
apto. 24, Cambuí, Campinas, SP, Brasil, CEP 13025-001.
E-mail: elisreginacosta @ yahoo.com.br. / evely@ unicamp.br
Trabalho adaptado da tese de doutorado da primeira autora,
realizada sob orientação da segunda autora. Projeto parcial-
mente financiado pela Coordenação de Aperfeiçoamento de
Pessoal de Nível Superior (Capes).
As autoras agradecem o apoio financeiro da Capes e do Conselho
Nacional de Desenvolvimento Científico e Tecnológico (CNPq). para uma prova. Alunos com baixo rendimento escolar normalmente utilizam estratégias ineficientes, acreditam que são menos capazes ou menos inteligentes do que os outros e, em geral, possuem a crença de que não há nada que possa ser feito para incrementar suas capacidades cognitivas (Zimmerman, 1998). À medida que os alunos com desempenho acadêmico baixo avançam na escola, suas dificuldades para aprender vão-se tornando cada vez maiores, piorando expressivamente o seu desempenho escolar.

Nas últimas décadas, pesquisadores e educadores têm-se dedicado a investigar qual a forma mais eficiente e adequada de se ensinar as estratégias de aprendizagem aos alunos, principalmente àqueles com dificuldades de aprendizagem (Bui, Schumaker, \& Deshler, 2006; Pressley et al., 1995). Propostas de intervenção em estratégias de aprendizagem têm-se mostrado extremamente eficientes, não só em suprir deficiências dos estudantes em diversas áreas acadêmicas, como matemática, leitura e escrita (Bui et al., 2006; Pressley et al.,1995), mas também em ajudar alunos a alcançarem níveis mais elevados de auto-regulação da sua aprendizagem, uma das principais metas da educação nos dias de hoje (Boruchovitch, 2004). 
Um modelo de intervenção que tem se mostrado válido para intervir e auxiliar crianças a superarem suas dificuldades de produção de textos e alcançarem um melhor desempenho em tarefas que envolvam a composição de textos narrativos, segundo Bui et al. (2006), é o de Desenvolvimento de Estratégias de Auto-Regulação na Escrita de MacArthur, Graham e Schwartz (1993).

Mais precisamente, no Modelo MacArthur et al. (1993), ensina-se aos alunos estratégias específicas para planejar e rever textos, em combinação com procedimentos para regular e controlar os processos cognitivos presentes na escrita. Isto é possibilitado, primeiramente, pelo ensino explícito das estratégias, no qual o instrutor ou professor modela, explica e proporciona a assistência necessária para que o aluno use as estratégias de forma eficiente. Outra forma de apoio do modelo é viabilizada pelo desenvolvimento de estratégias de auto-regulação. Orientar os alunos no sentido de desenvolver um diálogo interno para direcionar e avaliar o uso das estratégias, bem como manter comportamentos desejáveis e desencorajar os indesejáveis, durante a realização das tarefas, é uma forma de se promover o desenvolvimento da auto-regulação (auto-instrução, auto-afirmação, auto-avaliação).

Graham, Harris e MacArthur (1993) enfatizam que são quatro as características que viabilizam uma efetiva implantação do Modelo de Auto-Regulação na escrita. Primeiro, a ênfase que é dada ao ensino explícito de estratégias de aprendizagem e de procedimentos de auto-regulação. Alunos com dificuldades de aprendizagem necessitam de um ensino mais estruturado, extensivo e com instruções mais explícitas do que aqueles que não apresentam problemas. Em segundo lugar, o modelo de autoregulação adapta-se às diferenças individuais de cada aluno, ou seja, diferenças individuais podem ser respeitadas. Uma terceira característica-chave deste modelo é a aprendizagem colaborativa entre professor e os alunos. Finalmente, este modelo é baseado em critérios, nos quais para se alcançar a auto-regulação, passa-se por estágios. Os alunos poderão transitar por estas etapas até que aprendam todos os passos.

Considerando a escassez de estudos nacionais, no sentido de verificar as contribuições que as intervenções, por meio da instrução em estratégias de aprendizagem, podem oferecer à aprendizagem e ao desempenho acadêmico de alunos brasileiros, o presente estudo tem, pois, como objetivo, avaliar a eficácia de uma intervenção em estratégias de aprendizagem na produção de textos, baseada no Modelo de Desenvolvimento de Estratégias de Auto-Regulação na Escrita - de MacArthur et al. (1993), numa amostra de estudantes da $6^{\mathrm{a}}$ série do ensino fundamental.

\section{Método}

Fazendo parte de uma pesquisa mais ampla (Costa, 2005), o trabalho de intervenção em estratégias de aprendizagem relativas à escrita foi desenvolvido a partir de um delineamento quase-experimental em 3 etapas: pré-teste, intervenção e pós-teste. Aventaram-se as hipóteses iniciais de que alunos que participariam da intervenção proposta produziriam textos de melhor qualidade do que alunos que não tivessem essa oportunidade (grupo-controle), bem como que os alunos do grupo experimental melhorariam o seu desempenho após a realização das sessões de intervenção.

Mais especificamente, buscou-se examinar se haveria melhora na qualidade dos textos quanto a três aspectos: estrutural (elementos básicos), níveis qualitativamente diferentes de articulação de idéias (categorias hierárquicas) e erros ortográficos. Analisou-se também se houve mudança na quantidade de linhas escritas pelos participantes da intervenção, quando comparados aos do grupo-controle.

\section{Caracterização da Amostra}

Os participantes da presente pesquisa foram alunos da $6^{\mathrm{a}}$ série do ensino fundamental de uma escola pública da cidade de Catalão, Goiás. As classes escolhidas para comporem os grupos controle e experimental foram sorteadas aleatoriamente.

O grupo-controle constituiu-se originalmente de 17 crianças, com a média de idade de 12.6 anos, das quais 12 (70.6\%) pertenciam ao gênero feminino e 5 (29.4\%) ao sexo masculino; do total, $15(88.2 \%)$ nunca haviam repetido uma série e a maioria, 9 deles (ou 52.9\%), se autoavaliou como possuindo um desempenho escolar regular.

Já o grupo experimental foi composto por 18 participantes, com média de idade de 13.3 anos, dos quais 8 (44.4\%) pertenciam ao gênero feminino e $10(55.6 \%)$ ao masculino. Em relação ao desempenho escolar, $61.1 \%$ (11) dos participantes se auto-avaliaram como possuindo um rendimento bom, e $27.8 \%$ (5), um rendimento regular.

\section{Instrumentos Aplicados e Materiais Utilizados no Pré e no Pós-Teste}

Produção de um Texto Narrativo. Foi solicitado aos participantes que escrevessem um texto narrativo individualmente, a partir de uma história lida pelo pesquisador. O propósito deste procedimento foi avaliar e comparar o desempenho dos alunos na produção de um texto narrativo, antes e após a intervenção ser realizada. Vale ressaltar que a mesma história foi lida no pré e pós-teste.

\section{Procedimentos para Coleta de Dados}

Inicialmente, a pesquisadora fez contato com várias escolas públicas da cidade de Catalão, Goiás, apresentando ao diretor e/ou ao coordenador pedagógico em questão os objetivos da pesquisa, verificando a disponibilidade da escola em participar do estudo. Optou-se por coletar os dados na região do estado de Goiás, em face da necessidade de se conhecer uma outra realidade brasileira, tão pouco investigada. Selecionaram-se duas escolas, que atendiam a uma clientela composta basicamente por crianças de nível socioeconômico baixo. Uma escola foi sorteada para realização do estudo-piloto, e a outra, para a coleta de dados.

A diretora e os professores foram informados acerca do caráter confidencial da pesquisa. Além disso, foi enfatizado que o horário de coleta de dados no estado de Goiás seria 
definido pela própria escola, a fim de interferir o mínimo possível na sua rotina. Aos alunos também foi assegurado o caráter confidencial do estudo, que sua participação no estudo não influenciaria de forma alguma em suas notas ou desempenho na escola, bem como sobre o caráter voluntário de sua participação. É importante ressaltar que todos os cuidados éticos tomados na presente pesquisa tiveram como base a resolução no 196/96 do Ministério da Saúde, com a qual estão em consonância.

As sessões de coleta de dados e a intervenção ocorreram em uma escola da cidade, com perfil socioeconômico muito semelhante ao da escola do estudo-piloto, que disponibilizou as aulas de religião e artes para a coleta de dados no pré e no pós-teste e para a realização das sessões de intervenção. Foram utilizadas quatro aulas para aplicar coletivamente os instrumentos, sendo duas para o pré-teste e duas para o pós-teste.

No primeiro dia, foi solicitado aos alunos que escrevessem um texto narrativo, tendo como base uma história, contada pela pesquisadora, a respeito de um incêndio. Esse procedimento foi adotado considerando-se as investigações anteriores que apontavam a necessidade de, primeiramente, contextualizar os temas, para que, posteriormente, os alunos escrevessem (Calkins, 2002; Silva \& Spinillo, 1998). Neste sentido, foi sugerido o mesmo tema para a produção do texto narrativo, no pré-teste e no pós-teste. Contudo, foi permitido que os participantes narrassem uma história de maneira diferente da contada, utilizando, caso quisessem, de diversas possibilidades para manifestarem sua criatividade pessoal. Foi-lhes enfatizado que se mantivessem fiéis ao tema "incêndio" e não ao conteúdo da história inicialmente contada. Esse procedimento foi adotado para possibilitar um exercício mais livre do uso das estratégias de aprendizagem, ensinadas durante a intervenção. O tamanho da história ficou a critério do participante e o tempo de duração para a elaboração da narrativa foi de 50 minutos (a duração normal de uma aula).

A coleta de dados do pré-teste, no grupo-controle e no experimental, transcorreu de maneira tranqüila, dentro do limite da aula (50 minutos), sem nenhuma interrupção. A intervenção em estratégias de aprendizagem para a produção de narrativas foi realizada, em seguida, pela primeira autora, no grupo experimental, num total de sete encontros de 50 minutos cada, durante três semanas. Nenhum tratamento foi ministrado no grupo-controle.

Uma semana após a realização da intervenção, foi feita a coleta de dados relativa ao pós-teste, aplicando-se os mesmos instrumentos empregados no pré-teste em ambos os grupos. Aos participantes do grupo experimental, foi solicitado ainda que fizessem uma avaliação individual sobre os pontos positivos e negativos da intervenção. Foram utilizados como critérios de exclusão: o aluno não responder a um dos instrumentos no pré e/ou no pós-teste e a baixa freqüência às sessões realizadas. Todos os instrumentos utilizados no estudo e os principais passos do modelo de intervenção em estratégias de aprendizagem foram pré-testados em um estudo-piloto, numa turma de $6^{a}$ série de uma escola vizinha, que não fez parte da amostra. Não foram feitas modificações na organização das sessões e instrumentos, como decorrência do estudo piloto.

Procedimentos Gerais Relativos às Sessões de Intervenção

A intervenção em estratégias de aprendizagem na produção de textos foi planejada conforme o modelo de autoregulação na escrita, para o gênero narrativo, proposto por MacArthur et al. (1993), adaptada para o presente estudo por Costa e Boruchovitch (2002). A instrução em estratégias foi pensada de forma a ter uma sequiência lógica na organização das estratégias a serem ensinadas em todas as sessões. Procurou-se, assim, assegurar um ensino progressivo e adequado à série e à idade dos alunos. Em todas as sessões, além de se ensinarem as estratégias de produção de textos (ensino explícito e auto-regulação), os aspectos motivacionais e afetivos também foram trabalhados, como recomenda a literatura da área à luz da Psicologia Cognitiva com base na Teoria do Processamento da Informação que mostra que as intervenções mais bem sucedidas são aquelas que envolvem não só o ensino das estratégias de aprendizagem específicas, mas também que incluem o apoio às variáveis afetivo-motivacionais que garantem a sua melhor utilização (Hattie, Biggs, \& Purdie, 1996). Neste sentido, como descrito em Costa (2005), as orientações motivacionais, as crenças de auto-eficácia e sobre inteligência, bem como as atribuições de causalidade dos estudantes foram também trabalhadas, no decorrer da intervenção.

\section{Procedimentos de Análise de Dados}

Apesar da seleção aleatória dos grupos para os tratamentos, foi feita uma análise de sua homogeneidade, no tempo "pré", quanto às variáveis de interesse, tais como: gênero, idade, repetência, aspectos estruturais da narrativa, nível de articulação de idéias, erros ortográficos e número de linhas escritas.

As produções escritas pelos alunos no pré e no pós-teste foram analisadas qualitativamente e classificadas de quatro formas diferentes: aspecto estrutural (elementos bási$\cos$ ), categorias hierárquicas (elementos básicos em conjunto com o nível de articulação de idéias), número de erros ortográficos e quantidade de linhas produzidas.

Para fins da análise de dados relativos aos aspectos estruturais, ou seja, relativos aos elementos básicos, utilizou-se o somatório de itens apresentados na narrativa do participante e trabalhados em uma das sessões de intervenção, a saber: introdução do assunto, personagens, ambiente, problema ou propósito, ação, conclusão e emoções. Cada item presente no texto produzido pelo aluno teve a equivalência de 1 ponto, podendo, nesta classificação, a pontuação do aluno variar de 0 a 7.

Uma análise adicional da narrativa dos participantes se constituiu de uma combinação do escore obtido por eles nos elementos básicos com o escore alcançado na análise do nível de articulação de idéias no texto, estimado por uma nota que variou de 1 a 4 , sendo 4 a melhor nota. Considerou-se a articulação de idéias à habilidade do aluno em integrar os elementos básicos de uma narrativa (personagens, cenários, tempo, enredo, problema, conclusão e 
emoções) com as idéias que ele pretendeu expressar e desenvolver no texto, estabelecendo nexos e evidenciando a continuidade entre as partes do texto (Ferrari, Bouffard, \& Rainville 1998). Pela análise de conteúdo das narrativas dos participantes, podem-se identificar 4 categorias hierárquicas: (a) categoria 1 - história estruturalmente incompleta e mal-articulada; (b) categoria 2 - história com estrutura incompleta, relativamente bem-articulada; (c) categoria 3 - história com estrutura completa, mal-articulada; (d) categoria 4 - história completa, bem-articulada. Cabe mencionar que, para aferição da pontuação dos participantes, as narrativas foram analisadas por três juízes independentes e o percentual de concordância obtido variou de $90 \%$ a $100 \%$.

Em relação à análise por linhas produzidas e erros ortográficos, a quantidade de linhas escritas e o número de erros cometidos no pré e no pós-teste foram calculados e comparados.

Para comparar as variáveis categóricas entre os grupos, em cada tempo, foi utilizado o teste Qui-quadrado ou, quando necessário, o teste exato de Fisher (presença de valores esperados menores que 5). As variáveis numéricas foram comparadas entre os dois grupos por meio do teste de MannWhitney, tendo em vista a não-normalidade de sua distribuição.

Para analisar a evolução dos escores entre os tempos e entre os grupos, foi utilizada a Análise de Variância
(ANOVA) para medidas repetidas. Na comparação entre os grupos, em cada tempo, foi utilizado o teste de Tukey e, na comparação das medidas entre os tempos para cada grupo, foi utilizado o teste de contraste por perfil. O nível de significância adotado para os testes estatísticos foi de 5\% $(p<0.05)$.

Como a análise de variância para medidas repetidas permite três tipos de comparações ao mesmo tempo (comparações intergrupos, intragrupos e análise do efeito da interação entre grupos e tempos), os resultados serão apresentados, levando em conta essas três possibilidades de comparação.

\section{Resultados}

As primeiras análises, realizadas com o objetivo de averiguar se haviam diferenças iniciais entre os grupos experimental e controle, mostraram que eles eram comparáveis em quase todas as variáveis de interesse, com exceção da média de idade, inferior no grupo controle $(Z=1.98$; $p=0.048)$ e do número de linhas $(Z=2.30 ; p=0.022)$, significativamente mais elevadas nesse grupo.

A Tabela 1 sintetiza os principais resultados encontrados no presente estudo no que diz respeito à comparação entre grupos e entre tempos e a Tabela 2 a complementa, apresentando dados adicionais no que se refere à análise comparativa da evolução dos grupos.

Tabela 1

Resultados das ANOVAS para Comparação entre Grupos e Tempos

\begin{tabular}{|c|c|c|c|}
\hline Variável Dependente & $\begin{array}{l}\text { Comparação } \\
\text { entre Grupos }^{\mathrm{a}}\end{array}$ & $\begin{array}{l}\text { Comparação } \\
\text { entre Tempos }\end{array}$ & $\begin{array}{l}\text { Efeito da Interação } \\
\text { Grupo vs Tempo }\end{array}$ \\
\hline $\begin{array}{l}\text { Nível de articulação } \\
\text { (categorias hierárquicas) }\end{array}$ & $\begin{array}{l}F_{(1,33)}=0.07 \\
p=0.798\end{array}$ & $\begin{array}{l}F_{(1,33)}=8.14 \\
p=0.007^{\mathrm{d}}\end{array}$ & $\begin{array}{l}F_{(1,33)}=8.14 \\
p=0.007^{\mathrm{d}}\end{array}$ \\
\hline Aspecto Estrutural & $\begin{array}{l}F_{(1,33)}=1.15 \\
p=0.292\end{array}$ & $\begin{array}{l}F_{(1,33)}=7.08 \\
p=0.012^{\mathrm{c}}\end{array}$ & $\begin{array}{l}F_{(1,33)}=1.16 \\
p=0.289\end{array}$ \\
\hline Número de Linhas & $\begin{array}{l}F_{(1,33)}=0.92 \\
p=0.344\end{array}$ & $\begin{array}{l}F_{(1,33)}=1.15 \\
p=0.291\end{array}$ & $\begin{array}{l}F_{(1,33)}=11.78 \\
p=0.002 \mathrm{e}\end{array}$ \\
\hline Erros Ortográficos/Linha & $\begin{array}{l}F_{(1,33)}=0.27 \\
p=0.609\end{array}$ & $\begin{array}{l}F_{(1,33)}=0.01 \\
p=0.946\end{array}$ & $\begin{array}{l}F_{(1,33)}=0.76 \\
p=0.389\end{array}$ \\
\hline
\end{tabular}

Notas. (a) comparação entre grupos controle e experimental; (b) comparação entre tempos "pré” e "pós"; (c) diferenças significativas entre tempos: aumento significativo entre os tempos para ambos os grupos; (d) diferenças significativas entre tempos: aumento significativo entre os tempos apenas para grupo experimental ( $p<0.001$; Teste de Perfil: Pré ${ }^{1}$ Pós); (e) diferenças significativas entre grupos: grupo-controle maior que grupo experimental no tempo "pré" ( $p<0.05$; teste de Tukey); diferenças significativas entre tempos: redução significativa entre os tempos apenas para grupo-controle ( $p=0.003$; Teste de Perfil: "Pré" 1 "Pós"); (f) diferenças significativas entre grupos: grupo-controle menor que grupo experimental no tempo "pós" ( $p<0.05$; teste de Tukey); diferenças significativas entre tempos: aumento significativo entre os tempos apenas para grupo experimental $(p=0.024$; Teste de Perfil: Pré ${ }^{1}$ Pós).

Constata-se que não houve diferença significativa em relação ao aspecto estrutural no grupo experimental e no controle, na comparação entre grupos $\left(F_{(1,33)}=1.15 ; p=0.292\right)$ e na interação grupo versus tempo $\left(F_{(1,33)}=1.16 ; p=0.289\right)$. Já na comparação entre tempos, houve uma diferença sig- nificativa para os dois grupos, com aumento significativo entre tempos para ambos os grupos $\left(F_{(1,33)}=7.08 ; p=0.012\right)$. Pode-se dizer que, antes de a intervenção ser realizada, os participantes dos dois grupos já possuíam uma compreensão clara dos elementos básicos que deveriam estar pre- 
sentes em uma narrativa. Nota-se que, com o passar do tempo, tal conhecimento se aprimorou nos integrantes dos dois grupos.

As produções textuais dos alunos também foram comparadas no pré e no pós-teste quanto ao nível de articulação das idéias, em conjunto com a presença dos elementos básicos da narrativa (categorias hierárquicas). A análise de variância para medidas repetidas (Tabela 1) demonstrou ausência de diferença significativa na comparação entre os grupos controle e experimental $\left(F_{(1,33)}=0.07 ; p=0.798\right)$. Contudo, os dados apontaram diferenças altamente significativas na comparação entre tempos $\left(F_{(1,33)}=8.14 ; p=0.007\right)$ e no efeito da interação grupo versus tempo $\left(F_{(1,33)}=8.14\right.$; $p=0.007$ ) para o grupo experimental.

Um dos propósitos do presente estudo foi também verificar se crianças submetidas a uma intervenção em estratégias de produção de textos escreveriam uma quantidade maior de linhas por texto. Ao se analisar o desempenho dos grupos no pré-teste, o Teste de Mann-Whitney mostrou que, nesta variável, os grupos controle e experimental não eram comparáveis $(Z=2,30 ; p=0.02)$. Mais especificamente, os integrantes do grupo-controle escreveram textos com uma quantidade significativamente maior de linhas do que os alunos do grupo experimental, no tempo "pré".

A Tabela 1, apresentada inicialmente, mostra a ausência de diferença significativa em relação ao número de linhas escritas no grupo experimental e no controle, na comparação entre grupos $\left(F_{(1,33)}=0.92 ; p=0.344\right)$ e na comparação entre tempos $\left(F_{(1,33)}=1.15 ; p=0.291\right)$. Já ao se analisar a interação grupo versus tempo, observou-se uma redução altamente significativa entre os tempos para o grupo-controle $\left(F_{(1,33)}=11.78 ; p=0.002\right)$.

Um outro objetivo da presente investigação foi verificar se, nos textos produzidos pelos participantes, haveria diminuição dos erros ortográficos, como decorrência da intervenção em estratégias de produção de textos. Vale esclarecer que a ortografia, a pontuação e a gramática não foram aspectos trabalhados durante a intervenção.

A análise de variância para medidas repetidas, apresentada anteriormente, na Tabela 1, aponta a ausência de diferença significativa para os grupos controle e experimental, na comparação entre grupos $\left(F_{(1,33)}=0.27 ; p=0.609\right)$, entre tempos $\left(F_{(1,33)}=0.01 ; p=0.946\right)$ e na interação grupo versus tempo $\left(F_{(1,33)}=0.76 ; p=0.389\right)$, nos grupos experimental e controle, no que diz respeito aos textos produzidos conforme a análise por erros ortográficos.

Tabela 2

Análise Comparativa da Evolução dos Grupos Controle e Experimental quanto ao Aspecto Estrutural, às Categorias Hierárquicas, ao Número de Linhas e aos Erros Ortográficos

\begin{tabular}{|c|c|c|c|}
\hline Aspecto estrutural $^{\mathrm{a}}$ & G. Controle & G. Experimental & Total \\
\hline Redução & $5(29.41 \%)$ & $0(0.00 \%)$ & 5 \\
\hline Estável & $6(35.29 \%)$ & $9(50.0 \%)$ & 15 \\
\hline Aumento & $6(35.29 \%)$ & $9(50.0 \%)$ & 15 \\
\hline Total & 17 & 18 & 35 \\
\hline Nível de articulação (categorias hierárquicas) & G. Controle & G. Experimental & Total \\
\hline Redução & $4(23.53 \%)$ & $O(0.00 \%)$ & 4 \\
\hline Estável & $8(47.06 \%)$ & $7(38.89 \%)$ & 15 \\
\hline Aumento & $5(29.41 \%)$ & $11(61.11 \%)$ & 16 \\
\hline Total & 17 & 18 & 35 \\
\hline Número de linhas ${ }^{\mathrm{b}}$ & G. Controle & G. Experimental & Total \\
\hline Redução & $12(70.59 \%)$ & $6(33.33 \%)$ & 18 \\
\hline Estável & $1(5.88 \%)$ & $0(0.00 \%)$ & 1 \\
\hline Aumento & $4(23.53 \%)$ & $12(66.11 \%)$ & 12 \\
\hline Total & 17 & 18 & 35 \\
\hline Erros ortográficos $^{\mathrm{a}}$ & G. Controle & G. Experimental & Total \\
\hline Redução & $6(35.29 \%)$ & $12(66.67 \%)$ & 18 \\
\hline Aumento & $11(64.71 \%)$ & $6(33.33 \%)$ & 17 \\
\hline Total & 17 & 18 & 35 \\
\hline
\end{tabular}

Nota. a- Teste Exato de Fischer; b- Qui-quadrado; $p<0.05$.

A partir dos dados da Tabela 2, percebe-se que 50\% (9) do grupo experimental e $35.29 \%$ (6) do grupo-controle melhoraram seu conhecimento a respeito dos elementos básicos constituintes de uma narrativa. Outro dado interessante foi que não houve piora no desempenho por parte dos participantes do grupo experimental, o mesmo não ocorrendo no grupo-controle. Entretanto, o teste exato de Fisher revelou que esses achados somente aproximaram a significância estatística $(p=0.057)$. 
Tendo como base os dados da Tabela 2 e a ausência de diferenças significativas entre os grupos no tempo pré, quanto ao desempenho na escrita de textos, pode-se dizer que ocorreu uma mudança qualitativa expressiva no grupo experimental, já que $11(61,1 \%)$ participantes, num total de $18(100 \%)$ desse grupo, passaram de uma categoria inferior para uma superior; 38,8\% (7) alcançaram o nível máximo (categoria 4), e nenhum piorou. Já no grupo-controle, dos 17 alunos, somente $5(29,4 \%)$ apresentaram mudança de uma categoria inferior para uma superior; $23,5 \%$ (4) passaram de uma categoria superior para uma inferior, e 47\% (8) se mantiveram na mesma categoria. Esses dados foram confirmados, ao se analisar a evolução do desempenho dos alunos do pré para o pós-teste por meio do teste exato de Fisher ( $p=0.043$ ).

É importante destacar que, apesar de o grupo experimental ter mostrado uma evolução quanto ao número de linhas escritas no pós-teste, houve apenas uma equiparação e não uma superação deste em relação ao grupo-controle nesta variável. Estes dados se confirmam, ao se analisar a evolução do desempenho dos alunos do pré para o pós-teste, por meio do teste exato de Fisher $(p=0.018)$. Contudo, ao se analisar a evolução do desempenho dos alunos do pré para o pós-teste, por meio do teste Qui-quadrado, os dados mostraram uma tendência à significância estatística marginal $\left(\chi^{2}(1)=3.45 ; p=0.063\right)$ entre os tempos somente no grupo experimental. Cabe mencionar que foi feita uma análise de covariância, ajustando os erros ortográficos por linha, tendo em vista a diferença inicialmente encontrada entre os participantes, bem como o fato de que, quanto mais se escreve, maior é a probabilidade de se incorrer em erros ortográficos. Nota-se, na Tabela 2, a ocorrência de redução de erros ortográficos de $35.29 \%$ (6) de participantes do grupo-controle e $66.67 \%$ (12) de alunos do grupo experimental.

\section{Discussão}

O presente estudo partiu da hipótese de que alunos do grupo experimental produziriam textos de melhor qualidade do que os do grupo-controle, hipótese esta que foi parcialmente confirmada. Em linhas gerais, os resultados obtidos apontam que o ensino de estratégias de aprendizagem relativas à escrita auxiliou os alunos no desempenho de uma tarefa específica, como escrever textos narrativos. Pode-se dizer que os alunos que participaram da intervenção em estratégias de aprendizagem produziram textos no pós-teste de melhor qualidade, caracterizados por uma estrutura narrativa adequada, idéias mais bem articuladas, maior quantidade de linhas escritas e menos erros ortográficos. Esses dados são semelhantes aos encontrados por outras pesquisas, desenvolvidas no estrangeiro, nesta área, com estudantes da $4^{\mathrm{a}}, 5^{\mathrm{a}}, 6^{\mathrm{a}}$ e $7^{\mathrm{a}}$ séries (Bui et al., 2006; Kieft et al., 2006; Torrance et al., 2007). A consciência de que um texto narrativo deve conter alguns elementos essenciais, ou seja, deve possuir uma seqüência temporal de eventos, tais como introdução do tema, personagens, ação, tempo, lugar, entre outros aspectos, já era um conhecimento esperado para a idade e para a série cursada pelos alunos. Apesar disto, tal conhecimento foi trabalhado em uma das sessões realizadas, visto que a literatura e o modelo adotado para o presente estudo apontam que o foco central em uma intervenção para a produção de textos deve ser desenvolver os processos cognitivos mediante o ensino dos aspectos estruturais de um texto, do contexto social relacionado à escrita e do uso de estratégias de aprendizagem (Bui et al.,_2006)

No que diz respeito ao aspecto estrutural, ou seja, em relação à presença ou à ausência de elementos básicos, os estudantes que participaram da intervenção, em comparação com os que dela não fizeram parte, apresentaram um avanço considerável no conhecimento sobre os elementos básicos constituintes de uma narrativa. Entretanto, esta evolução não se mostrou estatisticamente significativa. É possível que este resultado se deva à amostra pequena, empregada no presente estudo.

Cabe mencionar que um bom texto narrativo não é composto somente por estrutura textual. A forma pela qual o aluno articula as idéias também é um aspecto a ser desenvolvido durante os anos escolares. Não se têm dúvidas de que as habilidades de antecipar perguntas da audiência e tornar claras as intenções do texto podem auxiliar sensivelmente na produção de textos. Neste sentido, os textos produzidos também foram analisados, tendo por base determinadas categorias hierárquicas, que combinavam a estrutura do texto e a articulação de idéias. Observou-se, no presente estudo, no transcorrer do pré para o pós-teste, uma mudança significativa de categorias entre os integrantes do grupo experimental. É possível que esse salto qualitativo dos participantes nas categorias hierárquicas possa ter sido fruto de um efeito da intervenção no que concerne a uma melhora produzida no nível de articulação das idéias.

Investigou-se, também, no presente estudo, a influência da intervenção na quantidade de linhas produzidas. Benefícios maiores foram alcançados por alunos do grupo experimental. Contudo, é interessante ressaltar que, apesar de ter ocorrido uma evolução quanto ao número de linhas escritas no pós-teste, de fato, aconteceu uma equiparação, e não uma superação, por parte dos alunos do grupo experimental em relação ao grupo-controle. Aventa-se a hipótese de que tenha ocorrido, durante a intervenção, uma evolução no processo de metas comunicativas (Bereiter \& Scardamalia, 1987), na qual os alunos parecem ter utilizado o método de "transformar o conhecimento" e não somente "contar". Transformar o conhecimento exige um controle consciente sobre as partes do processo de produção de textos, no sentido de acessar, generalizar e organizar as informações que conhecem (MacArthur et al., 1993), e, por conseguinte, amplia a noção de como realizar trabalhos elaborativos e reflexivos. Dados semelhantes foram encontrados por Calkins (2002), Ferrari et al. (1998) e La Paz e Graham (1997).

A pesquisa de La Paz e Graham (1997) indicou que o planejamento antecipado em alunos da $5^{\mathrm{a}}, 6^{\mathrm{a}}$ e $7^{\mathrm{a}}$ séries favorece a produção de textos mais longos, com maior coesão e qualidade. Segundo Calkins (2002), nessas sé- 
ries, espera-se que o processo de produção textual se torne mais complexo, isto é, que os alunos antecipem os finais dos parágrafos e as conclusões do texto, pensando em um maior número de possibilidades para escrever, já que, nesta etapa de escolarização, é esperado um domínio das convenções do padrão formal da língua portuguesa. Entretanto, as redações dos alunos da $6^{\mathrm{a}}$ série do grupo experimental do presente estudo não foram longas, nem apresentaram um nível de coesão e qualidade esperado para esta etapa da escolarização.

Uma investigação conduzida por Ferrari et al. (1998) para examinar o processo de escrita de universitários demonstrou que os estudantes mais eficientes produzem textos mais longos do que os considerados pouco eficientes. Um estudo desenvolvido por Graham, Harris e Mason (2005) mostrou que o ensino de estratégias de autoregulação proporcionou aos alunos da $3^{\text {a }}$ série a composição de textos mais longos, completos e qualitativamente melhores, em comparação com seus colegas. Se, por um lado, é sabido que nem sem sempre escrever mais é escrever melhor, por outro, a partir das pesquisas relatadas anteriormente, pode-se perceber que é também possível que o aumento na quantidade de linhas escritas seja acompanhado de um ganho qualitativo na produção, como a análise das categorias hierárquicas do presente estudo possibilitou constatar.

Torrance, Thomas e Robsinson (2000) afirmam que um outro fator que contribui para a produção de um texto de maior qualidade é a familiaridade do autor em relação ao conteúdo do tópico a ser escrito. De fato, pode-se acrescentar que não é somente o conhecimento do assunto e do gênero textual que distingue alunos que possuem maior ou menor habilidade na produção de textos, mas também o uso eficiente de estratégias de aprendizagem. Embora a familiaridade com o conteúdo seja importante para a qualidade da narrativa, tanto o grupo-controle quanto o experimental tiveram acesso a tarefas que lhes eram familiares, mas a qualidade e a melhora na produção de textos (na análise pelas categorias hierárquicas) se evidenciaram somente no grupo experimental, o que pode ser considerado indicativo da eficácia da intervenção. Recomenda-se, pois, que futuras pesquisas explorem melhor as relações entre o número de linhas, a qualidade do texto e o emprego de estratégias de aprendizagem adequadas ao escrever.

Um outro aspecto também avaliado no presente estudo foi se, nos textos produzidos pelos participantes, haveria diminuição dos erros ortográficos, como decorrência da intervenção realizada. Os dados mostraram uma tendência à diminuição dos erros ortográficos por parte dos estudantes do grupo experimental. Segundo Pressley et al. (1995), ao contrário do que se pode imaginar, a ênfase excessiva do professor nesses aspectos, ou a forte preocupação do aluno a esse respeito, pode prejudicar ou desencorajar o esforço de produzir textos mais sofisticados e de revisá-los depois.

É importante ressaltar que, lamentavelmente, a ausência de estudos nacionais abordando o tema da presente pesquisa, neste grau de escolaridade e utilizando o modelo adotado neste estudo, tornou as comparações e os diálogos com a literatura brasileira mais difíceis de serem realizados. Tendo em vista os resultados promissores e encorajadores encontrados em uma intervenção de curta duração, de caráter inédito, em nosso meio, recomenda-se que outros estudos sejam desenvolvidos neste enfoque. Acredita-se que os resultados obtidos na presente investigação possam abrir novas perspectivas de análise do processo de produção de textos, especificamente da narrativa, ampliando a compreensão, em nosso meio, dos modelos de intervenção na produção de textos propostos pelos teóricos cognitivistas, baseados na teoria do processamento da informação.

Cabe, entretanto, algumas considerações quanto às limitações do presente estudo, isto é, não há como descartar que possa ter havido efeito de testagem no pré- teste sobre o pós-teste. Estudos posteriores deveriam incluir uma classe não participante a ser testada somente no momento pós para uma averiguação mais precisa desse efeito. Outras medidas de controle das mudanças que podem ter emergido como decorrentes do desenvolvimento humano devem também ser pensadas e incorporadas às novas investigações.

Espera-se que as possíveis limitações na aplicação do modelo utilizado no presente estudo sejam verificadas e refinadas em pesquisas futuras, nas quais se possa não só ampliar a duração da intervenção, mas também voltá-la para outras séries, faixas etárias e grupos específicos de alunos, como, por exemplo, estudantes com dificuldade de aprendizagem.

Conclui-se pela necessidade de assegurar aos professores um conhecimento amplo sobre propostas de intervenção em estratégias de aprendizagem. É essencial que os cursos de formação de futuros professores os capacitem a analisar, ensinar e promover o uso de estratégias de aprendizagem adequadas em sala de aula (Boruchovitch, Costa, \& Neves, 2005). Boruchovitch et al. (2005) consideram que as contribuições da Psicologia Cognitiva baseada na Teoria do Processamento da Informação ainda não foram suficientemente difundidas e assimiladas de maneira prática nos cursos voltados para a formação de futuros professores.

\section{Referências}

Bereiter, C., \& Scardamalia, M. (1987). The psychology of written composition. Hillsdalle, NJ: Lawrence Erlbaum.

Boruchovitch, E. (2004). A auto-regulação da aprendizagem e a escolarização inicial. In E. Boruchovitch \& J. A. Bzuneck (Eds.), Aprendizagem: Processos psicológicos e o contexto social na escola (pp. 37-60). Petrópolis, RJ: Vozes.

Boruchovitch, E., Costa, E. R., \& Neves, E. R. C. (2005). Estratégias de aprendizagem: Contribuições para a formação de professores nos cursos superiores. In M. C. Jolly, A. A. A. Santos, \& F. F. Sisto (Eds.), Questões do cotidiano universitário (pp. 239-260). São Paulo, SP: Casa do Psicólogo.

Bui, Y. N., Schumaker, J. B., \& Deshler, D. D. (2006). The effects of a strategic writing program for students with and without learning disabilities in inclusive fifth-grade classes. Learning Disabilities Research e Practice, 21(4), 244-260. 
Calkins, L. M. (2002). A arte de ensinar a escrever: O desenvolvimento do discurso escrito. Porto Alegre, RS: Artes Médicas.

Costa, E. R. (2005). A intervenção por meio do ensino de estratégias de aprendizagem: contribuições para a produção de textos. Tese de Doutorado não-publicada, Universidade Estadual de Campinas, SP.

Costa, E. R., \& Boruchovitch, E. (2002). Adaptação de um programa para intervenção em estratégias de aprendizagem para textos narrativos. Manuscrito não-publicado.

Da Silva, A. L., \& De Sá, L. (1997). Saber estudar e estudar para saber. Porto, Portugal: Porto.

Ferrari, M., Bouffard, T., \& Rainville, L. (1998). What makes a good writer? Differences in good and poor writer's selfregulation of writing. Instructional Science, 26, 473-488.

Graham, S., Harris, K. R., \& Mac Arthur, C. (1993). Improving the writing of students with learning problems: Self-regulated strategy development. School Psychology Review, 22(4), 657669.

Graham, S., Harris, K. R., \& Mason, L. (2005). Improving the writing performance, knowledge, and self-efficacy of struggling young writers: The effects of self-regulated strategy development. Contemporary Educational Psychology, 30(2), 207-241.

Hattie, J., Biggs, J., \& Purdie, N. (1996). Effects of learning skill interventions on students learning: A meta-analysis. Review of Educational Research, 66(2), 99-136.

Kieft, M., Rijlaarsdam, G., \& Van den Bergh, H. (2006). Writing as a learning tool: Testing the role of students' writing strategies. European Journal of Psychology of Education, 21(1), 17-34.

La Paz, S., \& Graham, S. (1997). Effects of dictation and advance planning instruction on the learning problems. Journal of Educational Psychology, 89(2), 203-222.

Lopes, M. C. C. (1997). O uso de estratégias cognitivas $e$ metacognitivas no ensino aprendizagem da leitura de $1^{\circ}$ grau: Uma proposta de intervenção. Dissertação de Mestrado nãopublicada, Universidade Estadual de Campinas, SP.

MacArthur, C., Graham, S., \& Schwartz, S. (1993). Integrating strategy instruction and word processing into a process approach to writing instruction. School Psychology Review, 23(4), 671-681.

Pozo, J. I. (1995). Estratégias de aprendizagem. In C. Coll, J. Palácios, \& A. Marchesi (Eds.), Desenvolvimento psicológico e educação: Psicologia da educação (Vol. 2, pp. 176-197). Porto Alegre, RS: Artes Médicas.

Pressley, M., Woloshyn, V., Burkell, J., Cariglia-Bull, T., Lysnchunk, L., McGoldrick, J. A., et al. (1995). Cognitive strategy instruction that really improves children's academic performance. Cambridge, MA: Brookline Books.

Silva, M. E. L., \& Spinillo, A. G. (1998). Uma análise comparativa da escrita de histórias pelos alunos de escolas públicas e particulares. Revista Brasileira de Estudos Pedagógicos, 193(79), 5-16.

Torrance, M., Fidalgo, R., \& Garcia, J. (2007). The teachability and effectiveness of cognitive self-regulation in sixth-grade writers. Learning and Instruction, 17(3), 265-285.

Torrance, M., Thomas, G. V., \& Robinson, E. J. (2000). Individual differences in undergraduate essay writing strategies: A longitudinal study. Higler Education, 39, 181-200.

Zimmerman, B. J. (1998). Academic studying and development of personal skill: A self-regulatory perspective. Educational Psychologist, 33(2/3), 73-86. 